\title{
Hydrogen sulfide prevents apoptosis of human PMN via inhibition of p38 and caspase 3
}

\author{
Laura Rinaldi ${ }^{1}$, Giuliana Gobbi ${ }^{1}$, Maurizia Pambianco ${ }^{2}$, Cristina Micheloni ${ }^{1}$, \\ Prisco Mirandola ${ }^{1}$ and Marco Vitale ${ }^{1,3}$ \\ ${ }^{1}$ Department of Anatomy, Pharmacology \& Forensic Medicine, Human Anatomy Section, University of \\ Parma, Ospedale Maggiore, Parma, Italy; ${ }^{2}$ Terme di Sirmione, Cell Biology Laboratory, Terme di Sirmione, \\ Brescia, Italy and ${ }^{3}$ ITOI-CNR, Bologna Unit, c/o Research Institute 'Codivilla-Putti', IOR, Bologna, Italy
}

\begin{abstract}
Hydrogen sulfide, together with carbon monoxide and nitric oxide, is now considered a gasotransmitter able to induce specific cellular responses. As hydrogen sulfide is a component of several natural compounds known to be effective in many inflammatory pathologies, particularly of the respiratory tract, we studied its effects in vitro

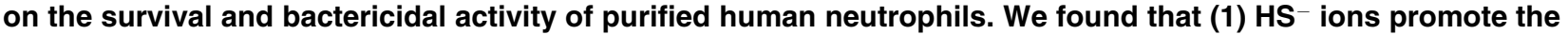
survival of granulocytes, but not that of lymphocytes or eosinophils, cultured in serum-free medium; (2) the pro-survival effect of $\mathrm{HS}^{-}$is due to inhibition of caspase-3 cleavage and p38 MAP kinase phosphorylation; (3) the bactericidal activity of neutrophils is not impaired by hydrogen sulfide. We conclude that $\mathrm{HS}^{-}$promotes $^{-}$ the short-term survival of neutrophils potentially accelerating the resolution of inflammatory processes and preventing the occurrence of new ones.
\end{abstract}

Laboratory Investigation (2006) 86, 391-397. doi:10.1038/labinvest.3700391; published online 30 January 2006

Keywords: hydrogen sulfide; apoptosis; granulocytes; thermalism; respiratory tract; thermal waters

Hydrogen sulfide $\left(\mathrm{H}_{2} \mathrm{~S}\right)$ has long been known as a toxic gas. At high concentrations, $\mathrm{H}_{2} \mathrm{~S}$ forms a complex with the $\mathrm{Fe}^{3+}$ of mitochondrial cytochrome oxidase, consequently inhibiting cellular oxidative metabolism. ${ }^{1}$ Recently, however, it has emerged that $\mathrm{H}_{2} \mathrm{~S}$ is also endogenously generated from cysteine, in reactions catalyzed by cystathionine $\beta$-synthase (CBS) and cystathionine $\gamma$-lyase (CSE). ${ }^{2-4}$ Mounting data on endogenously generated $\mathrm{H}_{2} \mathrm{~S}$ have now included this gas in the family of gasotransmitters, together with nitric oxide (NO) and carbon monoxide (CO), and its effects start to be understood both at the cellular and molecular level. ${ }^{5}$ While a physiological role in hippocampal neurons longterm potentiation has also been demonstrated, ${ }^{6}$ perhaps the best characterized physiological effect of $\mathrm{H}_{2} \mathrm{~S}$ to date is the relaxation of vascular smooth muscle cells, generated by $\mathrm{H}_{2} \mathrm{~S}$-mediated $K_{\text {АтP }}$ channel opening. ${ }^{7}$

Correspondence: Dr M Vitale, MD, Department of Anatomy, Pharmacology \& Forensic Medicine, University of Parma, Ospedale Maggiore, Via Gramsci, 14, Parma I-43100, Italy.

E-mail: marco.vitale@unipr.it

Received 26 October 2005; revised 15 December 2005; accepted 16 December 2005; published online 30 January 2006
Interestingly, it has recently been demonstrated that a decreased production of endogenous $\mathrm{H}_{2} \mathrm{~S}$ is involved in the pathophysiology of hypoxic hypertension in rats and that exogenous administration of $\mathrm{H}_{2} \mathrm{~S}$ can exert beneficial effects in this pathology. ${ }^{8,9}$ Smooth muscle relaxant effects of $\mathrm{H}_{2} \mathrm{~S}$ have also been demonstrated to have a physiological role in the control of intestinal contractility. ${ }^{10}$ In vitro, $\mathrm{H}_{2} \mathrm{~S}$ has been demonstrated to induce serum-independent proliferation of nontransformed epithelial cells ${ }^{11}$ as well as apoptosis of human aorta smooth muscle cells via extracellular regulated kinase (ERK) induction. ${ }^{12}$ Moreover, it is well known that several natural compounds containing hydrogen sulfideas, for instance, sulfurous thermal waters-have clinically evident anti-inflammatory properties, particularly for the respiratory system and skin. The reducing properties of $\mathrm{HS}^{-}$ions are known, in fact, to have an antibacterial potential and a solubilizing effect on exocrine secretions of the upper respiratory tract that-in turn-restore a physiological muco-ciliary clearance. ${ }^{13}$ Of note, some time ago Valitutti and co-workers reported a dose-dependent inhibitory effect of sulfidecontaining waters on mitogen-induced T-lymphocyte proliferation and IL-2 production. ${ }^{14}$ 
Neutrophils are short-lived cells that are essential in the inflammatory response of tissues and organs. Aging neutrophils-both in the bloodstream and in the inflammatory tissue sites-are eliminated by apoptosis, with subsequent phagocytosis by macrophages and other bystander phagocytic cells. ${ }^{15}$ When cultured in vitro, most neutrophils die by spontaneous apoptosis within $24 \mathrm{~h}$. Several factors, however, can modulate the timing of neutrophils apoptosis in vitro, as exposure to stress stimuli (ie UV irradiation, osmotic stress, serum deprivation), or to apoptosis-inducing agents like Fas. ${ }^{16}$ As found in most cell types, caspases are the major executors of the neutrophil apoptotic program; members of the MAP kinase family, and specifically p38 MAPK, have been involved in stress-induced but not in spontaneous neutrophil apoptosis. Two signal transduction pathways have, in fact, been demonstrated ${ }^{16}$ in neutrophils' apoptosis, both ultimately involving caspases. The first pathway-p38 MAPK independent-appears to be involved in spontaneous or Fas-mediated apoptosis; the second-p38 MAPK dependent but JNK/ERK1-2 independent-rather mediates stress-activated neutrophil apoptosis.

Given this complex background, we investigated the in vitro effects of hydrogen sulfide on human neutrophil apoptosis and its molecular basis in terms of signal transduction modulation.

\section{Materials and methods}

\section{PMN Purification}

Blood was collected from buffy coats of healthy donors. Purified human granulocytes were obtained by a two-step gradient separation. Briefly, $25 \mathrm{ml}$ of 1:4 PBS-diluted blood was layered upon $20 \mathrm{ml}$ of Lympholyte-H (Cedarlane, Hornby, Ontario, Canada) and centrifuged at $390 \mathrm{~g}$ for $35 \mathrm{~min}$. Plasma and platelets were discarded, while lymphocytes were collected for subsequent experiments in this step. The pellet of red cells and granulocytes was resuspended in cold lysis buffer $\left(\mathrm{NH}_{4} \mathrm{Cl} 155 \mathrm{mM}\right.$ and $\mathrm{KHCO}_{3} 10 \mathrm{mM}$ ) and centrifuged at $250 \mathrm{~g}$ for $10 \mathrm{~min}$. Recovered cells were then resuspended in PBS and the granulocytes were isolated from red-cell ghosts by a Percoll gradient (Sigma-Aldrich, Milano, Italy). In detail, $35 \mathrm{ml}$ of resuspended granulocytes were stratified on $15 \mathrm{ml}$ of a 1:1.58 Percoll PBS-dilution and centrifuged at $350 \mathrm{~g}$ for 25 min. Purity of PMN cells was checked by anti-CD15 FITC mAb (Exalpha, Maynard, MA, USA) staining and flow cytometry. Only samples exceeding 95\% purity were used for subsequent experiments. Contaminant lymphocytes were also monitored by staining PMN aliquots with anti-CD3 FITC mAb (Exalpha).

In some experiments, eosinophils were isolated from granulocytes preparations by immunomagnetic negative selection based on a anti-human CD16 antibodies cocktail (Milteny, Auburn, CA, USA).
Purity of eosinophils was assessed by immunofluorescent double staining with anti-CD15 FITC and anti-CD16 PE mAbs (Exalpha) and flow cytometry.

\section{Cell Cultures and Treatment}

Purified human PMN or lymphocytes were cultured up to $24 \mathrm{~h}$, at an optimal cell density of $1 \times 10^{6} \mathrm{cell} /$ $\mathrm{ml}$, in serum-free RPMI medium (Cederlane). NaHS (Aldrich, cat. no. 161527) was immediately added to the cell cultures at concentrations ranging from 0.23 to $3.66 \mathrm{mM}$. Control cultures were treated as above in the absence of NaHS.

In some experiments, purified cell cultures were treated for $24 \mathrm{~h}$ with (i) the MEK-1/MEK-2 inhibitor U0126 $(30 \mu \mathrm{M})$, or (ii) the PI3 kinase inhibitor LY294002 $(60 \mu \mathrm{M})$, or (iii) the p38 MAPK inhibitor SB202190 $(30 \mu \mathrm{M})$. Caspase activation was inhibited by ZVAD-FMK $(30 \mu \mathrm{M})$. All inhibitors were from Calbiochem (Darmstadt, Germany), and were resuspended in DMSO stock solution. Control samples were therefore treated with DMSO alone at the same working dilutions. Cell cultures were treated with the appropriate inhibitor $30 \mathrm{~min}$ before addition of NaHS. The NaHS solution was prepared immediately before use at $1 \mathrm{M}$ concentration in RPMI medium, and added to the cell cultures to the final concentration indicated. ${ }^{17}$

\section{Assessment of Apoptosis}

Cell culture viability was assessed by trypan blue exclusion. Apoptotic cells were analyzed by flow cytometry by staining unfixed cells with propidium iodide (PI) $(2.5 \mu \mathrm{g} / \mathrm{ml}$ in PBS). The percentage of dead/apoptotic cells was monitored by flow cytometry gating on PI-positive cells. Apoptotic cells stained dimly with PI, while necrotic cells stained bright, as previously described. ${ }^{18}$

\section{Western Blot}

Cultured cells $\left(1 \times 10^{6}\right)$ were collected at specific time points, washed in PBS and centrifuged at $200 \mathrm{~g}$ for $10 \mathrm{~min}$. Pellets were resuspended in Laemmli sample buffer $(0.125 \mathrm{M}$ Tris-HCl pH 6.8, SDS $8 \%$, glycerol $48 \% \mathrm{w} / \mathrm{v}, 2$-mercaptoethanol $20 \% \mathrm{v} / \mathrm{v}$ and blue bromophenol). The samples were heated at $95^{\circ} \mathrm{C}$ for $5 \mathrm{~min}$ run on $10 \%$ SDS-acrylamide gels and finally blotted onto nitrocellulose filters.

Blotted filters were blocked and incubated with specific primary antibodies following manufacturers' protocols. Rabbit polyclonal anti-phosphop38, anti-Caspase-3, and anti-phospho-PDK-1 antibodies (Cell Signaling Technology, Beverly, MA, USA) were all used at the concentration of $1 \mu \mathrm{l} / \mathrm{ml}$. Mouse anti- $\beta$-actin (Sigma) was diluted $1: 5000$.

Filters were then washed and further incubated for $1 \mathrm{~h}$ at room temperature with 1:5000 peroxidase- 
conjugated anti-rabbit or with 1:2000 peroxidaseconjugated anti-mouse IgG (Pierce, Milwaukee, WI, USA) in the primary antibody working solution at room temperature. Specific reactions were revealed with the ECL Supersignal West Pico Chemiluminescent Substrate detection system (Pierce).

\section{Bactericidal In Vitro Assay}

Bacteria used in this study were a clone of Escherichia coli HB101 strain (Takara Bi Inc., Otsu, Japan), stably transformed for Kanamycin resistance. Bacteria were kept in log phase of growth in Luria broth (LB), in the presence of $30 \mu \mathrm{g} / \mathrm{ml}$ Kanamycin (Sigma) and stored in $10 \%$ glycerol at $-80^{\circ} \mathrm{C}$. Stationary phase overnight cultures were transferred to $1 \mathrm{ml}$ of RPMI medium (E. coli dilutions were $1: 200,1: 20000,1: 2000000$ ) without antibiotic or serum and grown overnight at $37^{\circ} \mathrm{C}$ with or without
$4 \times 10^{6}$ of human granulocytes cultured for $24 \mathrm{~h}$ in the presence of $1.83 \mathrm{mM}$ NaHS. E. coli concentration was determined in the supernatants as OD absorbance measured by a spectrophotometer (Anthos 2010; Duotech s.r.l., Milano, Italy) at $620 \mathrm{~nm}$ wavelength. To determine exactly the residual viability of bacteria, aliquots of $E$. coli/PMN supernatants were dispersed in LB-agar-kanamycin plates. The number of $E$. coli colonies was then counted after overnight growth at $37^{\circ} \mathrm{C}$.

The number of $E$. coli remaining alive in $1 \mu \mathrm{l}$ of the supernatant of $E$. coli/PMN cultures has been reported as colony forming units $(\mathrm{CFU}) / \mu \mathrm{l}$.

\section{Transmission Electron Microscopy}

Neutrophil cultures were pelleted at $200 \mathrm{~g}$ for $10 \mathrm{~min}$, washed and fixed in $2.5 \%$ glutaraldehyde in $0.1 \mathrm{M}$ PBS, $\mathrm{pH}$ 7.4. Pellets were then fixed in $2 \%$ osmic acid in $0.1 \mathrm{M} \mathrm{PB}, \mathrm{pH} 7.4$, and embedded in
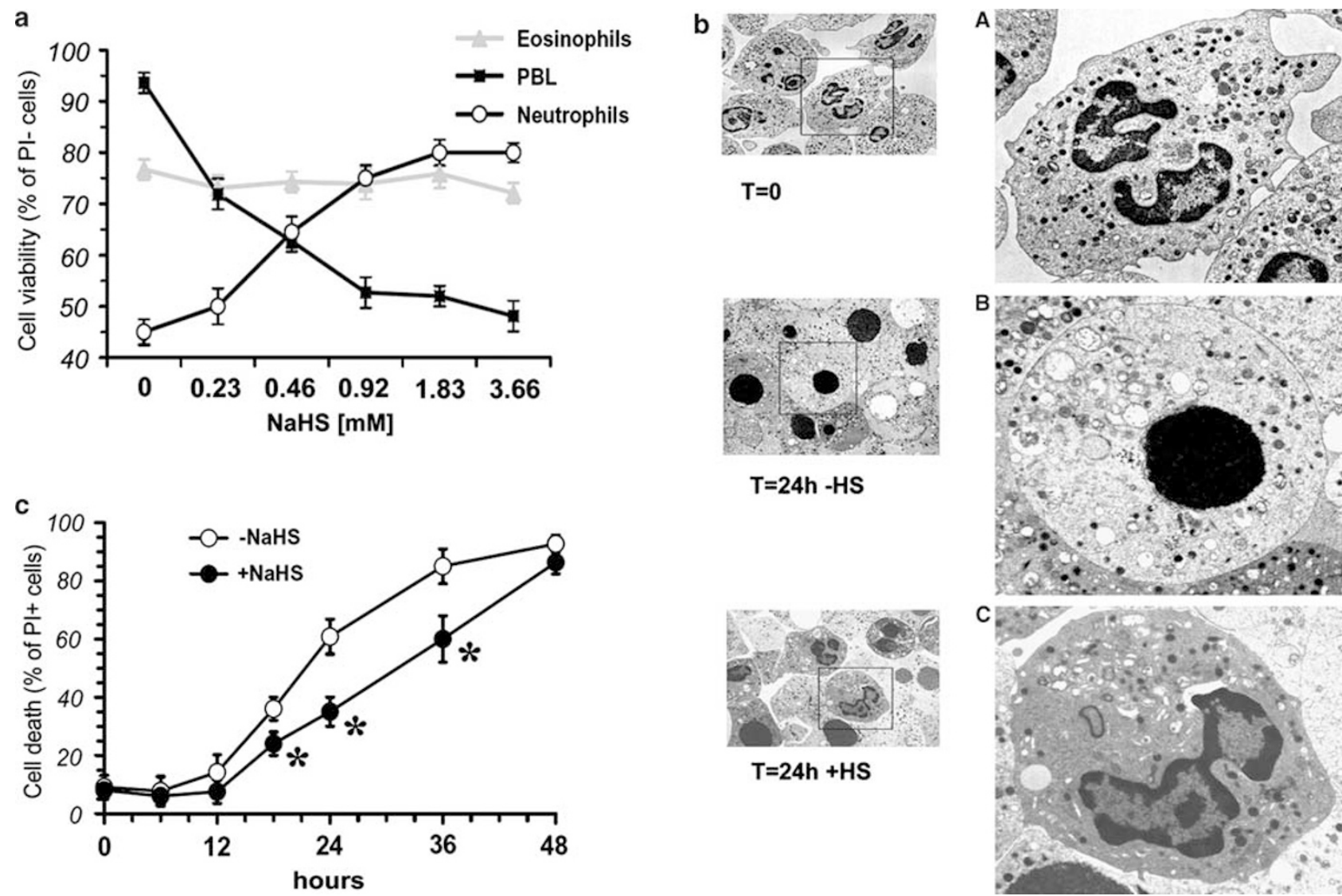

Figure 1 Effect of $\mathrm{HS}^{-}$on neutrophils viability in culture. (a) Viability of purified neutrophils, eosinophils and lymphocytes from the same subjects after $24 \mathrm{~h}$ culture in serum-free medium at increasing concentrations of NaHS. Viability was measured as percentage of PInegative cells, by flow cytometry. Means \pm s.e. of five independent experiments. (b) Transmission electron microscopy of neutrophils cultures immediately after purification $(T=0)$ or $24 \mathrm{~h}$ later $(T=24)$ in the presence $(+\mathrm{HS})$ or absence $(-\mathrm{HS})$ of $1.83 \mathrm{mM}$ NaHS. The three images on the left are shown at a magnification of $\times 3192$. Cells in the frames are enlarged in the right panels. At $T=0$, (A) most neutrophils are healthy. At $T=24$, in the absence of HS $^{-}$most neutrophils are apoptotic whereas in the presence of HS ${ }^{-}$the number of apoptotic cells decreases significantly. In (B) apoptotic cells show a typical morphology, with micronuclei and initial cytoplasmic vacuolization. (c) Time-course analysis of NaHS effects on PMN spontaneous cell death. PMN were treated (filled circles) with 1.83 mM NaHS and spontaneous cell death was quantified by flow cytometry at the indicated times. NaHS significantly prevents spontaneous PMN death at 18,24 and $36 \mathrm{~h} .{ }^{*} P<0.01$ compared to untreated cells at the same time points. Means \pm s.e. of three independent experiments. 
Durcupan (Fluka Chemie, Buchs, Switzerland). Semithin sections were stained with Toluidine blue- $0.5 \%$ sodium carbonate, while ultra-thin sections were stained with uranyl acetate and lead citrate according to Reynolds ${ }^{19}$ and observed with a Philips 300 electron microscope (Philips, Eindhoven, The Netherlands).

\section{Statistical Analysis}

All values in the figures and text are expressed as means \pm standard error of $n$ observations (with $n \geq 3$ ). Data sets were examined by analysis of variance (ANOVA) and Dunnett's test (when required). A $P$-value less than 0.05 was considered as statistically significant.

Dose-response curves of cell viability were analyzed with the nonlinear least squares curvi-fitting program Prism (GraphPAD, San Diego, CA, USA). Percentages of living cells at different doses of NaHS were fitted with a sigmoid dose-response function, thus obtaining the dose that produced a $50 \%$ reduction of spontaneous cell death $\left(\mathrm{EC}_{50}\right)$.

\section{Results}

\section{HS $^{-}$Selectively Promotes Neutrophils Viability}

The effects of hydrogen sulfide on PMN viability were assessed in vitro on purified human PMN cultures, supplemented with increasing doses of NaHS (ranging from 0 to $3.66 \mathrm{mM}$ ). Neutrophil viability was quantified after $24 \mathrm{~h}$ cultures by PI staining and flow cytometry (Figure 1a). Whereas in control cultures the viability was around $50 \%$, increasing doses of NaHS increased cell viability up to $80 \%$.

The NaHS dose that produced a $50 \%$ reduction of spontaneous cell death (EC50 \pm s.e.) was $0.50 \pm$ $0.12 \mathrm{mM}$. Transmission electron microscopy (TEM) analysis of purified neutrophils cultured $24 \mathrm{~h}$ in the presence/absence of NaHS showed that the morphology of viable cells in the presence of $\mathrm{HS}^{-}$was similar to that of freshly purified, healthy cells $(T=0)$. On the contrary, most cells in the absence of HS $^{-}$died by apoptosis (Figure 1b). Time-course experiments performed with a fixed dose of NaHS $(1.83 \mathrm{mM})$ showed that apoptosis of neutrophils was significantly inhibited between 18 and $36 \mathrm{~h}$ treatment (Figure 1c). As controls, we isolated from the same donors both eosinophils and PBL. Whereas NaHS at the same doses did not affect eosinophils' viability, it significantly enhanced PBL cell death. To exclude that small lymphocytes contaminants in purified PMN preparations might release factors potentially responsible for the observed pro-survival effect of $\mathrm{HS}^{-}$on neutrophils, we co-cultured PMN and lymphocytes from the same donor at different ratios. Figure 2 shows that lymphocyte co-culture did not modify the effects of $\mathrm{HS}^{-}$on PMNs.
To investigate the cell signalling pathway(s) involved in $\mathrm{HS}^{-}$pro-survival effect on granulocytes, we next treated our cell cultures with various pharmacological inhibitors that selectively interfere

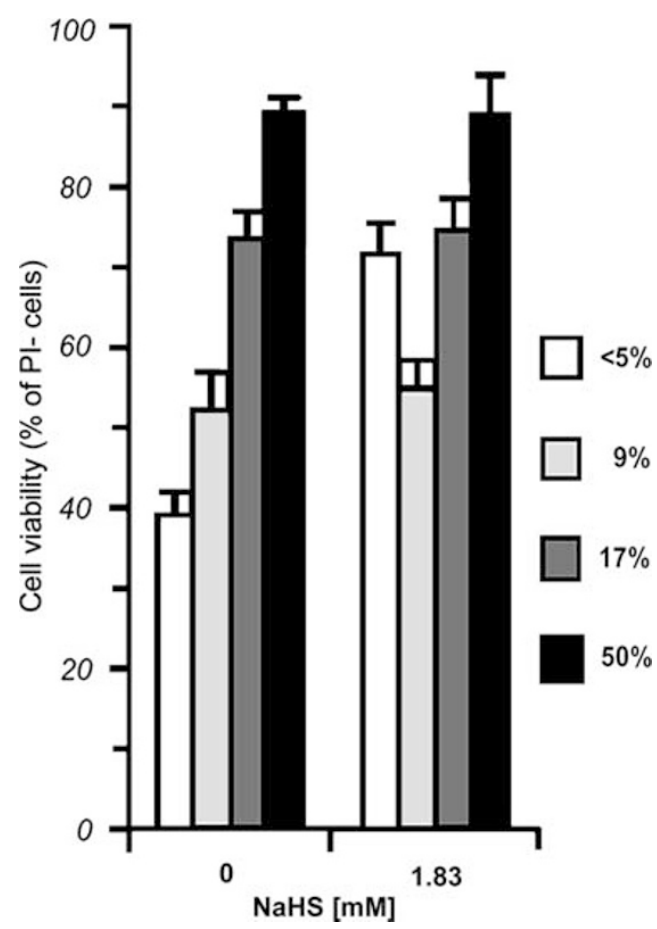

Figure 2 Effects of lymphocytes on PMN viability. Increasing numbers of autologous lymphocytes were cultured with neutrophils in the presence or absence of $1.83 \mathrm{mM}$ NaHS. Bars indicate the percentage of living neutrophils detected by flow cytometry as PI-negative cells. Means \pm s.e. of three independent experiments.

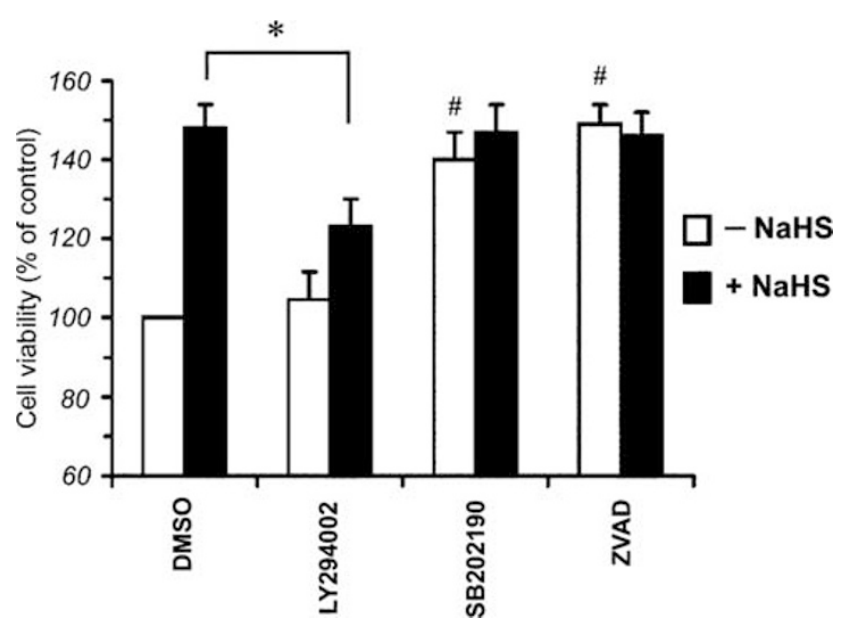

Figure 3 Effect of different pharmacological inhibitors on the survival of purified human neutrophils cultured for $24 \mathrm{~h}$ in the presence or absence of $1.83 \mathrm{mM}$ NaHS. Control samples were treated with DMSO alone. The PI3 kinase inhibitor LY294002 was used at a concentration of $60 \mu \mathrm{M}$; the p38 MAP kinase inhibitor SB202190 was used at a concentration of $30 \mu \mathrm{M}$, while the general caspase inhibitor ZVAD was used at a concentration of $30 \mu \mathrm{M}$. Means \pm s.e. of three independent experiments. ${ }^{*} P<0.01$ LY294002/NaHS-treated cells vs NaHS-treated control cells. ${ }^{\#} P<0.01$ SB202190 or ZVAD-treated cells vs untreated control (DMSO) cells. 
with PI3 kinase, MAP kinase and caspase activities. Figure 3 shows that ZVAD is able to completely preserve the viability of granulocytes after $24 \mathrm{~h}$. This result was somehow expected, because the death of granulocytes is essentially due to caspasedependent apoptosis. Similarly, the inhibition of p38 MAPK by SB202190 significantly increased granulocytes survival, like ZVAD and $\mathrm{HS}^{-}$alone. On the contrary, the PI3 kinase inhibitor LY294002 abrogated the pro-survival effect induced by $\mathrm{HS}^{-}$, not affecting the viability of cells cultured in the absence of $\mathrm{HS}^{-}$.

On the basis of these functional data, we therefore analyzed by Western blot the cleavage of caspase-3 and the phosphorylation of p38 MAP kinase in our granulocytes cultures in the presence or absence of NaHS. Figure 4 shows that NaHS $(1.83 \mathrm{mM})$ inhibits caspase-3 cleavage at $6 \mathrm{~h}$ as well as p38 MAP kinase phosphorylation as early as $1 \mathrm{~h}$, and that this inhibitory effect persists after $6 \mathrm{~h}$. Of note, $\beta$-actin degradation, which is an early event in granulocytes apoptosis, is also inhibited by NaHS.

\section{Bactericidal Effects of Granulocytes}

To understand whether or not the pro-survival effect of NaHS on granulocytes could impair their bactericidal activity, we finally performed a set of experiments in which granulocytes were co-cultured with E. coli HB101 in the presence or absence of NaHS. Figure 5 shows that neutrophils cultured in the presence of NaHS $(1.83 \mathrm{mM})$ were able to kill bacteria as efficiently as neutrophils in the absence of NaHS. After $24 \mathrm{~h}$, as expected, neutrophils cultured in the presence of NaHS are much more efficient then those cultured in the absence of NaHS.

\section{Discussion}

Hydrogen sulfide is now considered, together with $\mathrm{CO}$ and NO, a 'gasotransmitter' able to generate cellular responses upon specific induction. $\mathrm{H}_{2} \mathrm{~S}$ can be endogenously generated from cysteine or exogenously administered to cells, frequently as a a

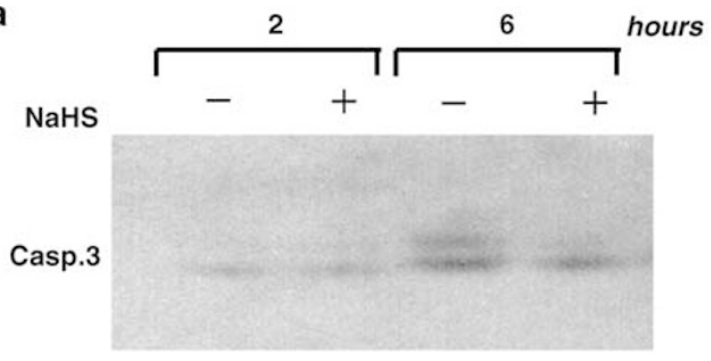

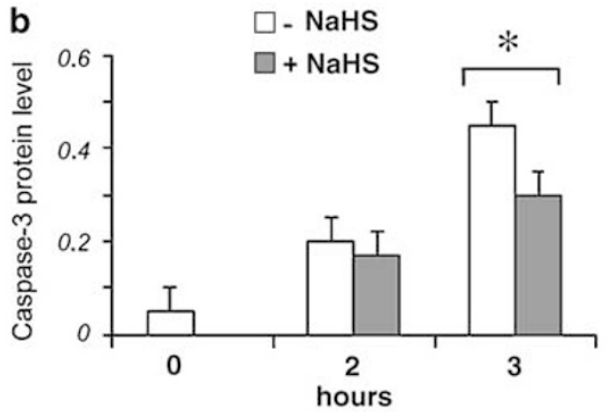

C

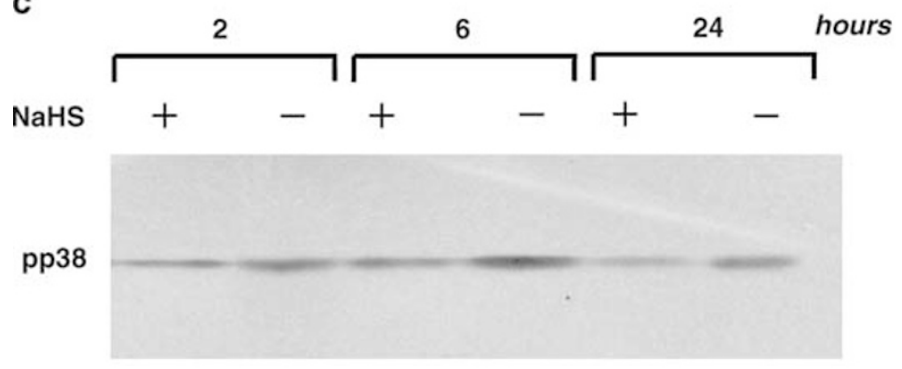

e

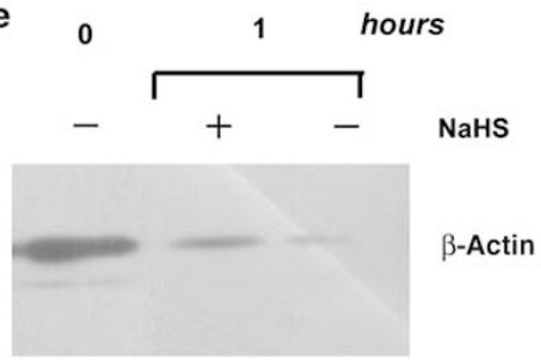

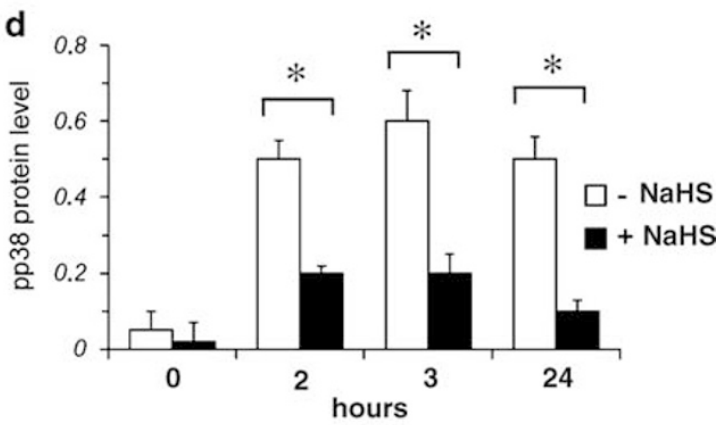

f

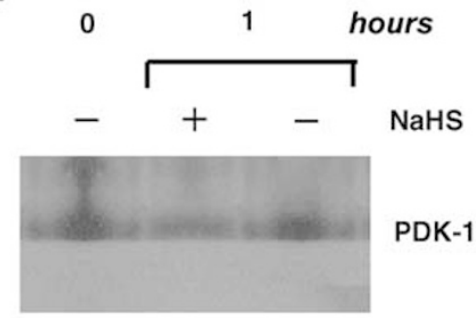

Figure 4 Time course of caspase 3 (a) and p38 MAP kinase (c) phosphorylation in the presence or absence of NaHS (1.83 mM) in purified neutrophils cultures. (b) and (d) the densitometric histograms of the respective Western blots. (e) The degradation of $\beta$-actin is inhibited by NaHS, while (f) no modulation of PDK-1 could be observed. Representative Western blots from three independent experiments are shown. Histograms are expressed as means \pm s.e. of three independent experiments. ${ }^{*} P<0.01$. 

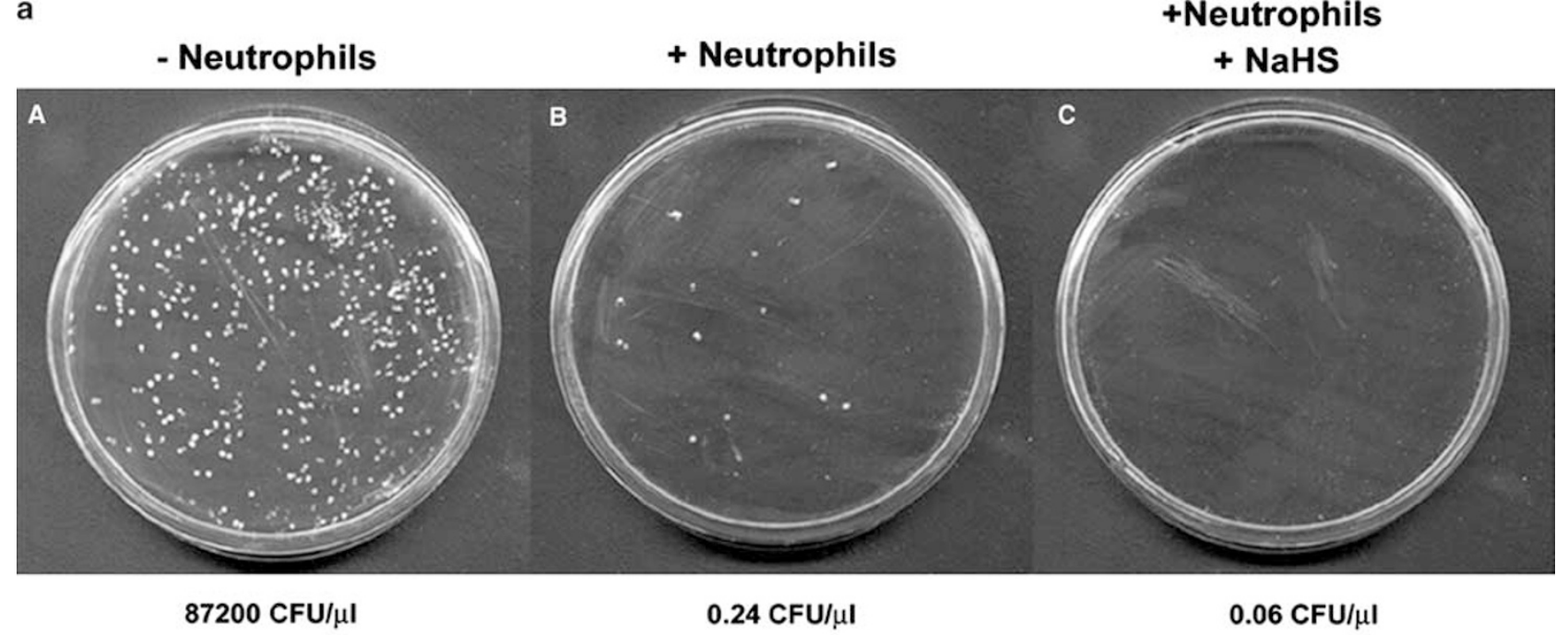

\section{+ Neutrophils \\ $+\mathrm{NaHS}$}

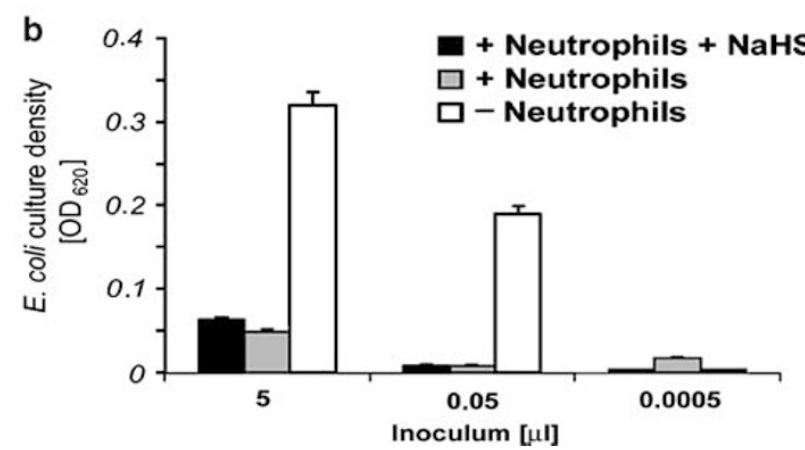

Figure 5 Bactericidal properties of neutrophils in the presence or absence of NaHS. (a) The presence of NaHS (1.83 mM) does not affect the bactericidal properties of neutrophils. On the contrary, no residual colony can be observed in the presence of NaHS (C) owing to the higher number of surviving neutrophils. (b) The densitometric histograms of the culture supernatants of three independent experiments. Means \pm s.e. of three independent experiments.

component of more complex sulfurous compounds, such as sulfurous thermal waters.

Knowledge about the effects of such compounds is currently confined to clinical information about their efficacy in the prevention and resolution of bronchial and pulmonary diseases characterized by mucous hypersecretion, inflammatory infiltrate and impairment of mucociliary clearance. Hypersecretion of mucus, in particular, is the defining feature of chronic bronchitis and is associated with an inflammatory process involving epithelium, glands and central airways. Of note, it has recently been demonstrated that progression of chronic obstructive pulmonary disease (COPD) is significantly associated with increasing infiltration of the airways by polymorphonuclear neutrophils and lymphocytes, ${ }^{20}$ which play a fundamental role against the development of microbial colonization and infection that worsen the progression of chronic bronchitis and COPD.

Given the lack of basic biological information on the effects of sulfur ions on the cellular subsets of the inflammatory infiltrate-as opposed to the abundance of clinical and epidemiological observa- tions on the beneficial effects of sulfur compounds-we decided to study in vitro the effects of sulfur ions on purified polymorphonuclear cell cultures.

We demonstrate that $\mathrm{HS}^{-}$ions have pro-survival effects on polymorphonuclear neutrophils in vitro, delaying the onset of apoptosis. Particularly under the stress condition of serum-deprivation, the prolonged survival of neutrophils in the presence of $\mathrm{HS}^{-}$is ultimately due to the inhibition of Caspase-3 cleavage, which delays the onset of apoptosis.

In fact, pharmacological inhibition of caspases prolongs the life of neutrophils, as do $\mathrm{HS}^{-}$ions. Frasch and co-workers ${ }^{16}$ described the spontaneous or Fas-induced neutrophil apoptosis as independent from p38 MAPK activity. While recent data from Alverado-Kristensson offer a deeper insight on the role of p38 in Fas-induced apoptosis, showing that p38 can directly phosphorylate and inhibit caspase 8 and $3,{ }^{16}$ a general agreement exists on the p38 MAPK dependence of stress-induced apoptosis of human neutrophils. Moreover, Yang et al recently demonstrated that both endogenously 
generated and exogenous $\mathrm{H}_{2} \mathrm{~S}$ can inhibit cell proliferation. ${ }^{21}$

Accordingly, our data show that sulfur ions inhibit p38 MAPK phosphorylation, which is relevant in inducing apoptosis in neutrophils, as pharmacological inhibition of p38 by SB202190 efficiently inhibits apoptosis to the same extent as HS $^{-}$ions. Moreover, surviving neutrophils show efficient bactericidal properties, which adds clear physiological relevance to their prolonged survival.

The pro-survival effect of $\mathrm{HS}^{-}$should be considered specific for neutrophils, as no effect was observed on eosinophils, and opposite effects were observed on lymphocytes of the same donors. These different biological effects might explain some contradictory data that can be found in the still scarce literature available on $\mathrm{HS}^{-}$. The opposite effects of sulfur ions on the viability of neutrophils vs lymphocytes might represent the basis for the potentiation of the acute inflammatory and bactericidal response and de-potentiation of the chronic and inflammatory cellular response. Such an inbalance of cellular infiltrate of the respiratory airways during inflammation-together with the known direct antibacterial and fluidifying effects on mucus-might likely represent the underlying biological mechanism of sulfurous water compounds that are successfully used in the prevention and therapy of chronic inflammatory diseases of the respiratory tract.

\section{Acknowledgements}

This work was supported by Federterme grants. We are grateful to Instrumentation Laboratory, Italy for technical support and to Vincenzo Palermo, Domenico Manfredi, Davide Dallatana and Luciana Cerasuolo for technical support.

\section{References}

1 Gosselin RE, Smith RP, Hodge HC. Clinical Toxicology of Commercial Products, 5th edn. Williams and Wilkins Ltd: Baltimore, USA, 1984.

2 Finkelstein JD, Martin JJ. Methionine metabolism in mammals. Distribution of homocysteine between competing pathways. J Biol Chem 1984;259:9508-9513.

3 Navarra P, Dello Russo C, Mancuso C, et al. Gaseous neuromodulators in the control of neuroendocrine stress axis. Ann NY Acad Sci 2000;917:638-646.

4 Moore PK, Bhatia M, Moochhala S. Hydrogen sulfide: from the smell of the past to the gas of the future? Trends Pharmacol Sci 2003;24:609-611.

5 Wang R. Two's company, three's a crowd: can $\mathrm{H}_{2} \mathrm{~S}$ be the third endogenous gaseous transmitter? FASEB J 2002;16:1792-1798.
6 Eto K, Ogasawara M, Umemura K, et al. Hydrogen sulfide is produced in response to neuronal excitation. J Neurosci 2002;22:3386-3391.

7 Zhao W, Zhang J, Lu Y, et al. The vasorelaxant effect of $\mathrm{H}_{2} \mathrm{~S}$ as a novel endogenous gaseous $K_{\text {ATP }}$ channel opener. EMBO J 2001;20:6008-6016.

8 Chunyu Z, Junbao D, Dingfang B, et al. The regulatory effect of hydrogen sulfide on hypoxic pulmonary hypertension in rats. Biochem Biophys Res Commun 2003;302:810-816.

9 Qingyou Z, Junbao D, Weijin Z, et al. Impact of hydrogen sulfide on carbon monoxide/heme oxygenase pathway in the pathogenesis of hypoxic pulmonary hypertension. Biochem Biophys Res Commun 2004;317:30-37.

10 Teague B, Asiedu S, Moore PK. The smooth muscle relaxant effect of hydrogen sulfide in vitro: evidence for a physiological role to control intestinal contractility. Br J Pharmacol 2002;137:139-145.

11 Deplancke B, Gaskins HR. Hydrogen sulfide induces serum-independent cell cycle entry in nontransformed rat intestinal epithelial cells. FASEB J 2003;17: 1310-1312.

12 Yang G, Sun X, Wang R. Hydrogen sulfide-induced apoptosis of human aorta smooth muscle cells via the activation of mitogen-activated protein kinases and caspase-3. FASEB J 2004;18:1782-1784.

13 Houtmeyers E, Gosselink R, Gayan-Ramirez G, et al. Regulation of mucociliary clearance in health and disease. Eur Respir J 1999;13:1177-1188.

14 Valitutti S, Castellino F, Musiani P. Effects of sulfurous (thermal) water on $\mathrm{T}$ lymphocyte proliferative response. Ann of Allergy 1990;65:463-468.

15 Savill JS, Wyllie AH, Henson JE, et al. Macrophage phagocytosis of aging neutrophils in inflammation. Programmed cell death in the neutrophil leads to its recognition by macrophages. J Clin Invest 1989;83: 865-875.

16 Frasch SC, Nick JA, Fadok VA, et al. p38 mitogenactivated protein kinase-dependent and -independent intracellular signal transduction pathways leading to apoptosis in human neutrophils. J Biol Chem 1998; 273:8389-8397.

17 Zhao W, Ndisang JF, Wang R. Modulation of endogenous production of $\mathrm{H}_{2} \mathrm{~S}$ in rat tissues. Can J Physiol Pharmacol 2003;81:848-853.

18 Hogg JC, Chu F, Utokaparch S, et al. The nature of small-airway obstruction in chronic obstructive pulmonary disease. N Engl J Med 2004;350:2645-2653.

19 Mirandola P, Ponti C, Gobbi G, et al. Activated human NK and CD8+ $\mathrm{T}$ cells express both TNF-related apoptosis-inducing ligand (TRAIL) and TRAIL receptors but are resistant to TRAIL-mediated cytotoxicity. Blood 2004;104:2418-2424.

20 Reynolds ES. The use of lead citrate at high $\mathrm{pH}$ as an electron-opaque stain in electron microscopy. J Cell Biol 1963;17:208-212.

21 Yang G, Cao K, Wu L, et al. Cystationine $\gamma$-lyase overexpression inhibits cell proliferation via a $\mathrm{H}_{2} \mathrm{~S}$ dependent modulation of ERK1/2 phosphorylation and p21 Cip/WAK-1. J Biol Chem 2004;279:49199-49205. 